\title{
Performance verification of the MIRI Imager flight model at CEA
}

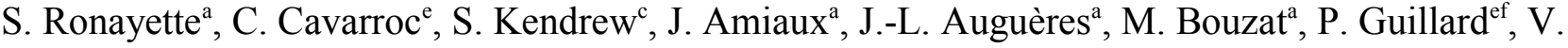

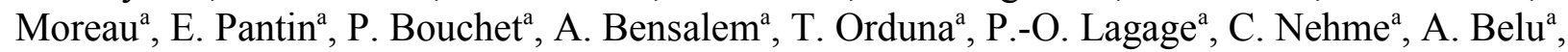 \\ A. Glasse ${ }^{\text {d }}$, P. Baudoz ${ }^{\text {b }}$ D. Dubreuil ${ }^{\mathrm{a}}$ \\ ${ }^{a}$ CEA, DSM, IRFU, SAp, bât. 709, Orme des Merisiers, 91191 Gif-sur-Yvette, France \\ ${ }^{\mathrm{b}}$ LESIA, Observatoire de Paris, 5 Place Jules Janssen, 92195 Meudon, France \\ ${ }^{\mathrm{c}}$ Leiden Observatory, PO Box 9513 , 2300 RA Leiden, The Netherlands \\ ${ }^{\mathrm{d}}$ UK Astronomy Technology Centre, Royal Observatory, Blackford Hill, Edinburgh EH9 3HJ, UK

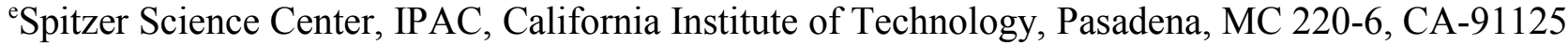 \\ ${ }^{\mathrm{f}}$ Institut d'Astrophysique Spatiale, CNRS/Université Paris Sud 11, Orsay, France
}

\begin{abstract}
MIRIM is the imager of the Mid Infrared Instrument (MIRI), one of the three scientific instruments on the James Webb Space Telescope (JWST). MIRIM will provide imaging between $5.6 \mu \mathrm{m}$ and $25.5 \mu \mathrm{m}$, low resolution spectroscopy (LRS) between 5 and $10 \mu \mathrm{m}$, and coronagraphy at $10.65 \mu \mathrm{m}, 11.4 \mu \mathrm{m}, 15.5 \mu \mathrm{m}$ and $23 \mu \mathrm{m}$.

The Optical bench Assembly of MIRIM Flight Model (FM) has been integrated and tested between 2008 and 2009 at CEA (Saclay, France). The tests consist in characterisation of optical performances at all wavelengths and in all three modes (imaging, spectroscopy and coronagraphy), using a test bench (or Ground Support Equipment - GSE) that has been developed for this purpose. The GSE comprises a helium cooled cryostat for the instrument itself, a proto-IR focal plane module (with JPL sensor chip and CEA electronics and housing), a warm telescope simulator that delivers a JWST-like beam, and computers and software for running automatic test procedures. It is designed to allow a large set of performance verifications, such as high-resolution PSF measurements, characterisation of coronagraphs, response to monochromatic line or resolving power of the spectroscopic mode, some of them being unique along the test program of the instrument.

After a short description of the test equipment, this paper focuses on the tests results. A full assessment of performances is given. When applicable, performances are cross checked with requirements.

Imaging mode and coronagraphy had already been validated on optically representative models along the MIRIM development plan, especially with the Engineering and Test Model (ETM) of MIRIM, early 2008. The FM test campaign allowed us to confirm that the flight model behaves as expected in these two modes. We also tested for the first time, and validated, the low-resolution spectroscopy mode.
\end{abstract}

Keywords : JWST, MIRI, infrared instrument, ground calibration, AIV, test bench.

\section{INTRODUCTION}

The Mid Infra Red Instrument (MIRI, Wright et al., 2004 ${ }^{[1]}$ ) is part of the scientific payload of the James Webb Space Telescope (JWST) and is built by a consortium of European institutes. It is divided in two subsystems: an integral field unit spectrometer and the MIRI Imager (MIRIM). MIRIM will provide imaging between $5.6 \mu \mathrm{m}$ and $25.5 \mu \mathrm{m}$, low resolution spectroscopy (LRS) between 5 and $10 \mu \mathrm{m}$, and coronagraphy at $10.65 \mu \mathrm{m}, 11.4 \mu \mathrm{m}, 15.5 \mu \mathrm{m}$ and $23 \mu \mathrm{m}$. This wide wavelength range associated to the three distinctive observation modes makes the ground testing and calibration of MIRIM challenging. The test bench at CEA was developed taking into account the restraints for coronagraphy, but it also allows thorough verification of the imaging and LRS modes.

The optical hardware for MIRIM includes five mirrors (four of which are powered), a cold stop for straylight control, a wheel with 18 filters and an 1024x1024 infrared detector with $25 \mu \mathrm{m}$ pixels. The design provides good pupil image, which is essential for coronagraphy, and uses a three-mirror anastigmate for beam focusing. It is free of chromatic aberration and provides diffraction limited beam at all wavelength in the $1.88 \times 1.88 \mathrm{arcmin}^{2}$ field of view. See figure 1.

Space Telescopes and Instrumentation 2010: Optical, Infrared, and Millimeter Wave, edited by Jacobus M. Oschmann Jr., Mark C. Clampin, Howard A. MacEwen, Proc. of SPIE Vol. $7731,77313 \mathrm{~N} \cdot$ ㅇ $2010 \mathrm{SPIE} \cdot \mathrm{CCC}$ code: 0277-786X/10/\$18 · doi: 10.1117/12.857125

Proc. of SPIE Vol. $773177313 \mathrm{~N}-1$ 


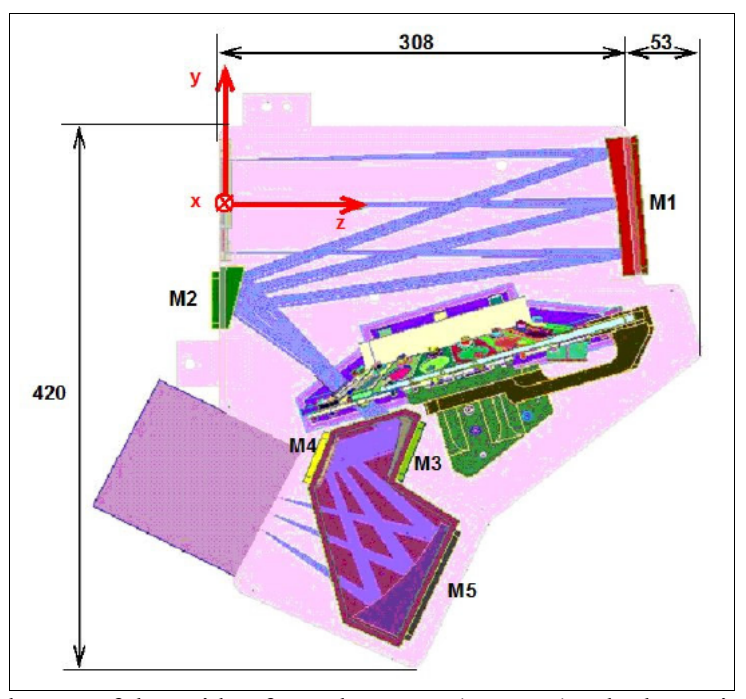

Figure 1. Optical and mechanical layout of the Mid-InfraRed IMager (MIRIM). The beam is first collimated by M1, redirected by M2 toward the filter wheel, and focused by the TMA (M3-M4-M5) on the detector (box on the bottom left)

The approach used for the full sequence of assembly, integration and verification of both the Verification Model and the Engineering and Test Model of MIRIM was proven to be successful and was applied to the Flight Model. This approach is presented in Amiaux et al., 2008[2]. The optical tests are performed sequentially in a well-defined order. First, the mirrors, a CCD camera and a pupil stop are mounted on the MIRIM structure. A grid of pinhole which simulates a star field is imaged on the CCD that can move in focus. The accurate location of the best focus is derived. Furthermore, this test allows a quick check at ambient temperature of image quality and measurement of magnification and distortion in the whole field of view. This "distortion grid" test is repeated at operating temperature $(7 \mathrm{~K})$. The filter wheel is then mounted and the alignment of the coronagraphic diaphragms is performed at ambient temperature. Then the alignment is verified at operating temperature. We then proceed to the next stage of performance verifications in all three modes, imaging, coronagraphy and low resolution spectroscopy.

This paper starts with the results of the first series of tests performed at both ambient and cryogenic temperatures: verification of image quality in section 2 and optical alignment in the following. Then the performance tests of the imaging mode at cryogenic temperature are detailed. The paper finishes with the results of the coronagraphy and low resolution spectroscopy tests. Each section starts with a brief description of the test set-up and method developed for the corresponding test.

\section{PRELIMINARY TESTS: IMAGE QUALITY}

\subsection{Overview and test set-up}

This test is the first to be done before going further in the test program. It is the first time that the full train of optics, from entrance focal plane to detector, is tested. It is a quick assessment of optical quality to check that all components are properly mounted and that the system does not suffer from misalignment, vignetting or defocus. We can measure basic optical characteristics (spot size, magnification, distortion) and we find the position at which the detector must be put in order to be at the best focus. For this purpose, we want to get in a single image the response of the instrument to a point-like source in the whole field of view and at visible wavelength. Tools have been developed in order to mount a grid of $87 \times 87$ five-micron pinholes, with a very regular pitch, at the entrance focal plane, illuminated by a LED system with a diffuser. On the exit focal plane, a CCD camera with $14 \mu \mathrm{m}$ pixels stores the image of the grid given by the instrument. The CCD camera is equipped with a micro-metric translation stage along the optical axis, which allows search for the best focus position. This is shown in figure 2 .

At cold temperature, the set-up is similar. A $7.7 \mu \mathrm{m}$ filter is fitted in the instrument. The grid step is larger to avoid overlap of the PSFs wings. The detector used is an engineering grade detector similar to the flight model one. It is fixed at the position found to be the best focus by the test at ambient. 


\subsection{Image quality and best focus at ambient temperature}

A portion of an image of the grid of pinholes is shown in figure 3. One can see the spot given by the nearly point-like sources formed by each hole of the grid $(5 \mu \mathrm{m}$ pinholes, reducing to a geometrical image smaller than $2 \mu \mathrm{m}$ in image plane). Note that holes of the grid are about 10 times smaller than the image of a point source given by MIRIM. Therefore, the width of the spots is mainly due to diffraction and geometrical aberrations. The latter are not negligible at visible wavelengths.

We have various criteria to determine the spot size. We considered the radius that encircles $65 \%$ of the energy contain in the PSF (R65). This criteria is measured in 9 area, or zones, regularly positioned in the field of view. The R65 of a given zone is the average R65 measured on all the spots found in the zone (about 100), and the uncertainty on the measurement is the standard deviation of the set of measurements. Each zone is small enough so that the image quality does not vary significantly across it. We give in figure 3 the results of this measurement as a function of the defocus. FWHM are also given.

At best focus we measure a R65 better than $15.5 \mu \mathrm{m}$ anywhere in the field of view. We showed, with numerical simulation, that this measure is systematically over-estimated by 2 to $3 \mu \mathrm{m}$ because of the under-sampling of the PSF (pixels of the CCD are $14 \mu \mathrm{m}$ large). We can conservatively assume a R65 of $14 \mu \mathrm{m}+/-1 \mu \mathrm{m}$ at best focus.

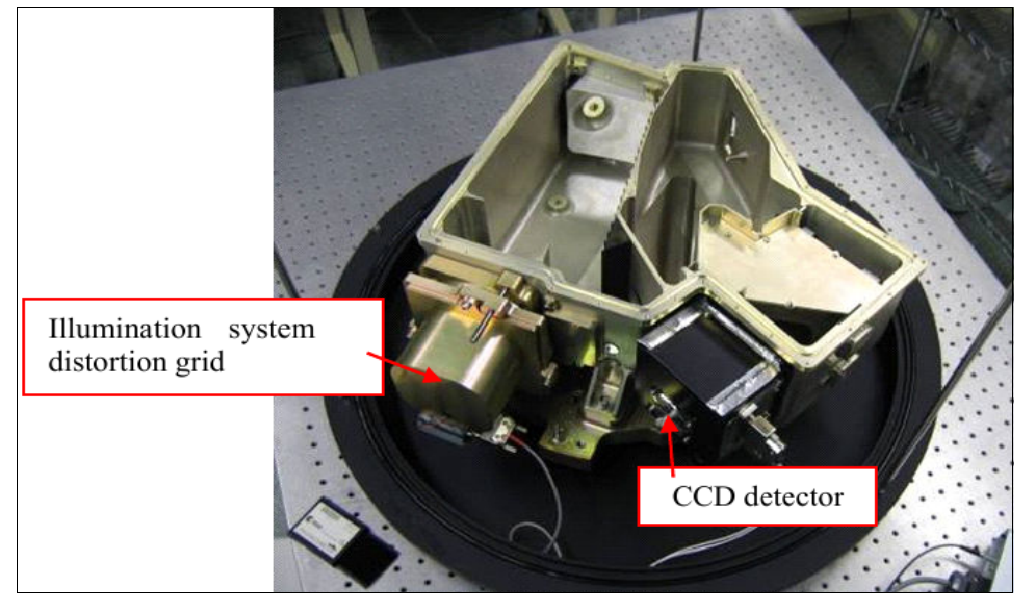

Figure 2: Set up for the optical quality test at ambient temperature.

We now want to compare these results with the specifications. Obviously, there are no specifications on encircled energy at visible wavelength. As a workaround, we compare our measurement with the optical model of the instrument. The following approach is used:

1 The criteria used for comparison is the R65 (readily computed by Zemax). A Zemax model describing the system under test (visible wavelength, no filter, ambient temperature) is built. Direct comparison with nominal design shows that our system is degraded from the nominal one. Mechanical tolerances must be applied (their effect on encircled energy is important at visible wavelength)

2 The Zemax model is toleranced, and a statistical study is done, using 500 Monte-Carlo trials. We know from the tolerance analysis that $98 \%$ of these MC trials give a system that is compliant with the specification on WFE at infrared wavelength.

3 The R65 at visible wavelength is computed for each of these MC trials. A statistical analysis gives the value of the R65 we can expect with a probability of $98 \%$, that we can call R6598\%

4 The statistical results are compared with our measure. If the measure is better than R65 ${ }_{98 \%}$, it means that the system is better than the worst Zemax model that is within specification, so it is considered within specification.

We have $\mathrm{R} 65_{98 \%}=16.2 \mu \mathrm{m}$. Therefore our system behaves as expected at visible wavelengths. 

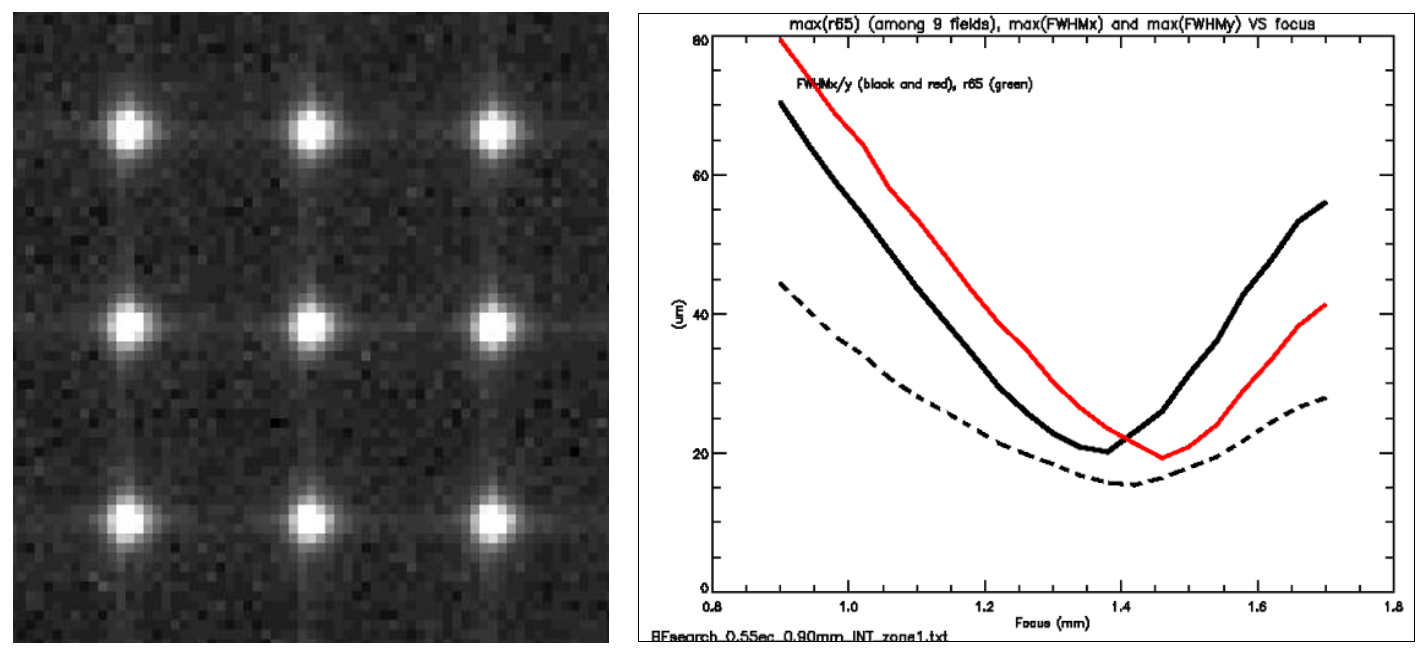

Figure 3. Left: details of spots given by distortion grid near best focus at $\lambda=635 \mathrm{~nm}$. Right: R65 (dash line) and FWHM X/Y (plain lines) vs Detector position at visible wavelength $(\lambda=635 \mathrm{~nm})$. The highest value in the field for each detector focus position is plotted. Uncertainty on R65 measurement is $+/-1 \mu \mathrm{m}$.

\subsection{Image quality at operating temperature}

Image quality must be verified at operating temperature $(<7 \mathrm{~K})$ and wavelengths. The all-aluminum design should ensure an homogeneous shrinking of the system and thus no change in performances. The filter chosen is the $7.7 \mu \mathrm{m}$ because it is the shortest wavelength that is Nyquist sampled.

Again, this system is not comparable to the nominal system, as far as the optics are concerned. Indeed, the pupil in this test is not a JWST-like pupil, but the imager diaphragm itself, which is larger. The approach to check that our results are compliant with the model is the same than before: a Zemax model is built, toleranced, and a statistical analysis is done on 500 Monte-Carlo trials.

The R65 measured is smaller than $40.2+/-1.3 \mu \mathrm{m}$ everywhere in the field. The R65 $98 \%$ is $41.5 \mu \mathrm{m}$. This is illustrated in figure 4 , where the measured and simulated encircled energies are plotted as a function of the 9 fields.

The MIRIM flight model behaves as expected in terms of image quality at operating temperatures.

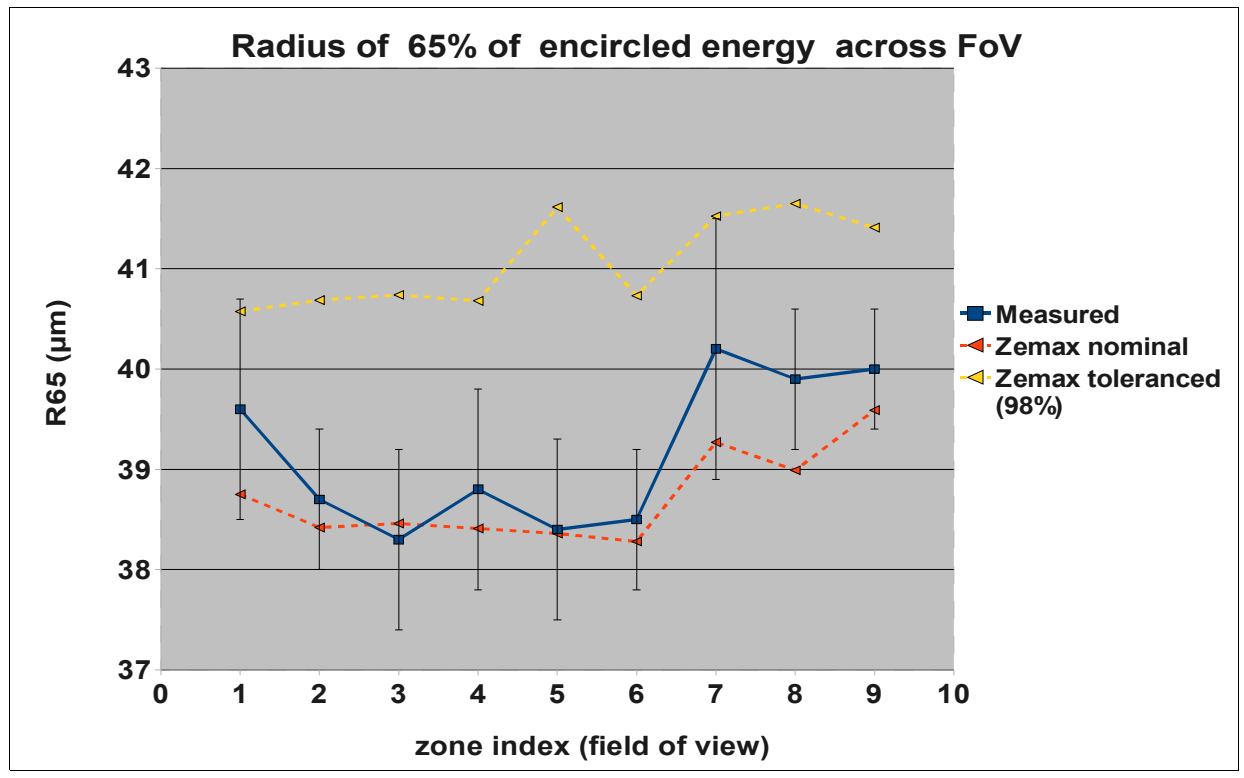

Figure 4. Comparison of measured and modeled encircled energies for the distortion grid cold test, for different part of the field of view 


\subsection{Other optical measurements}

\section{Magnification}

On the distortion grid images, we get the centroid of each spot with an accuracy estimated to $+/-0.5$ pixels. And we know from metrology that the grid is very accurate, i.e. we can consider that the pinholes are perfectly regularly spaced. We can then derive magnifications $\gamma_{\mathrm{x}}$ and $\gamma_{\mathrm{y}}$ in the $\mathrm{X}$ and $\mathrm{Y}$ direction respectively. The method leads to a very good accuracy, of $+/-0.4 \%$ on the value of magnification. The error is due to the error in estimating the centroid of each spot.

We measure a magnification of $0.352+0.008 /-0.000$ in the whole field of view. This is in good agreement with the Zemax model (0.352), and well within the specified magnification of $0.352+0.017 /-0.000$ that ensures a pixel field of view of 0.11 arcsec on sky.

\section{Distortion}

Distortion occurs when magnification varies across the field. We can measure distortion by comparing the predicted position of the spot on the detector array (position as if there were no distortion) with the real position.

We can compute the amount of distortion for each spot in the image, and create a map of distortion. This is shown in figure 5 below. The same map from the optical model is also shown. We can see that our system behaves as expected as far as the distortion is concerned.

Both distortion and magnification have been verified at operating temperature. Again, the test confirmed that there are no change in performances, as expected with such a design that ensures an homogeneous shrinking of the system.
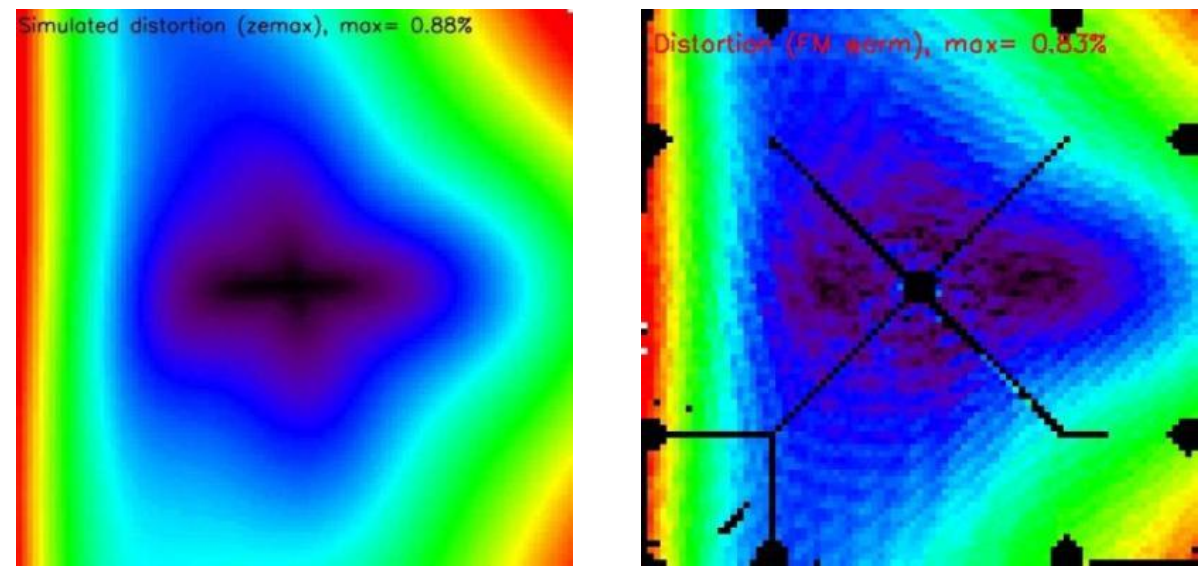

Figure 5. left: distortion as simulated by Zemax. Values range from 0 to $0.88 \%$. Right: measured distortion. Values range from 0 to $0.83 \%$

\section{ALIGNMENT}

\subsection{Overview and test set-up}

The alignment of MIRIM is the process by which the coronagraphic diaphragms (that lie in the pupil plane) are accurately centred on the MIRIM optical axis. Indeed, the tolerance analysis showed that, mechanically, it was not possible to obtain a sufficiently good alignment directly when assembling the parts and adjustment may be required. This is a very important step to ensure optimal performances of the coronagraphic observations.

The test consists in pointing the coronagraphic diaphragms with an alignment telescope that is co-aligned with MIRIM optical axis (figure 6). If the alignment requirement of the system is not fulfilled, a correction is applied to minimize the shift between the optical axis and the centre of the diaphragms.

To see the diaphragm through the alignment telescope, MIRIM entrance pupil must be re-imaged at infinity. This is done by the use of a lens (the alignment lens tool - ALT) mounted at the object focal plane of MIRIM.

The accuracy of the measurement is limited by various factors: positioning and mounting of the ALT, setting of the alignment telescope along MIRIM optical axis, measurement accuracy through the eyepiece. This accuracy is estimated 


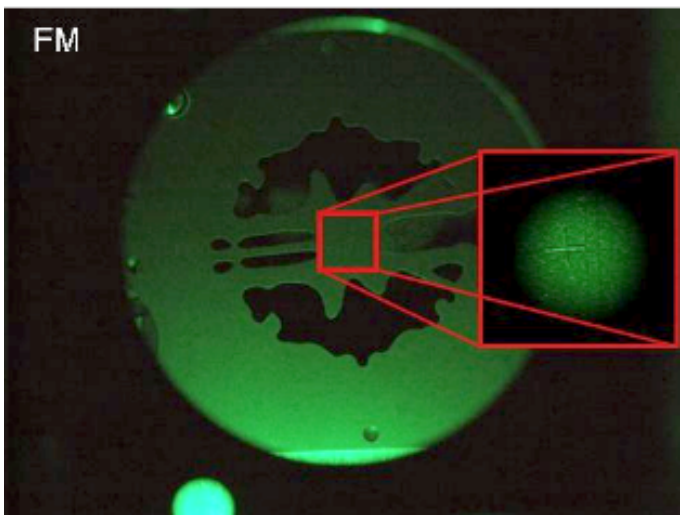

Figure 6. coronagraphic diaphragms as seen through the eyepiece of the alignment telescope. The alignment telescope is accurately aligned on MIRM optical axis. The centre of the diaphragm is materialized by an engrave cross and its position measured with respect to the optical axis.

to $+/-23 \mu \mathrm{m}$ (in term of distance in the diaphragm plane) at ambient temperature and to $+/-34 \mu \mathrm{m}$ for the test performed cold. The loss of accuracy when going cold is due to a change of reference surfaces when MIRIM is in the cryostat. This accuracy is sufficient anyhow. The specification is to centre the diaphragms on the optical axis with an accuracy of $+/-140 \mu \mathrm{m}$. The correction is done by placing shims of a given thickness on the fixation pads of M1, in order to steer the optical axis. The required shim is small enough so that image quality and field of view alignment are not deteriorated.

\subsection{Results}

Alignment has been done at ambient temperature before vibrations, then verified at operating temperature and after vibrations. Figure 7 illustrates the effect on M1 shimming on the coronagraphic diaphragm centring. The process brings all the diaphragm within the required $140 \mu \mathrm{m}$.

The final position of the diaphragms is given in table 1 below.

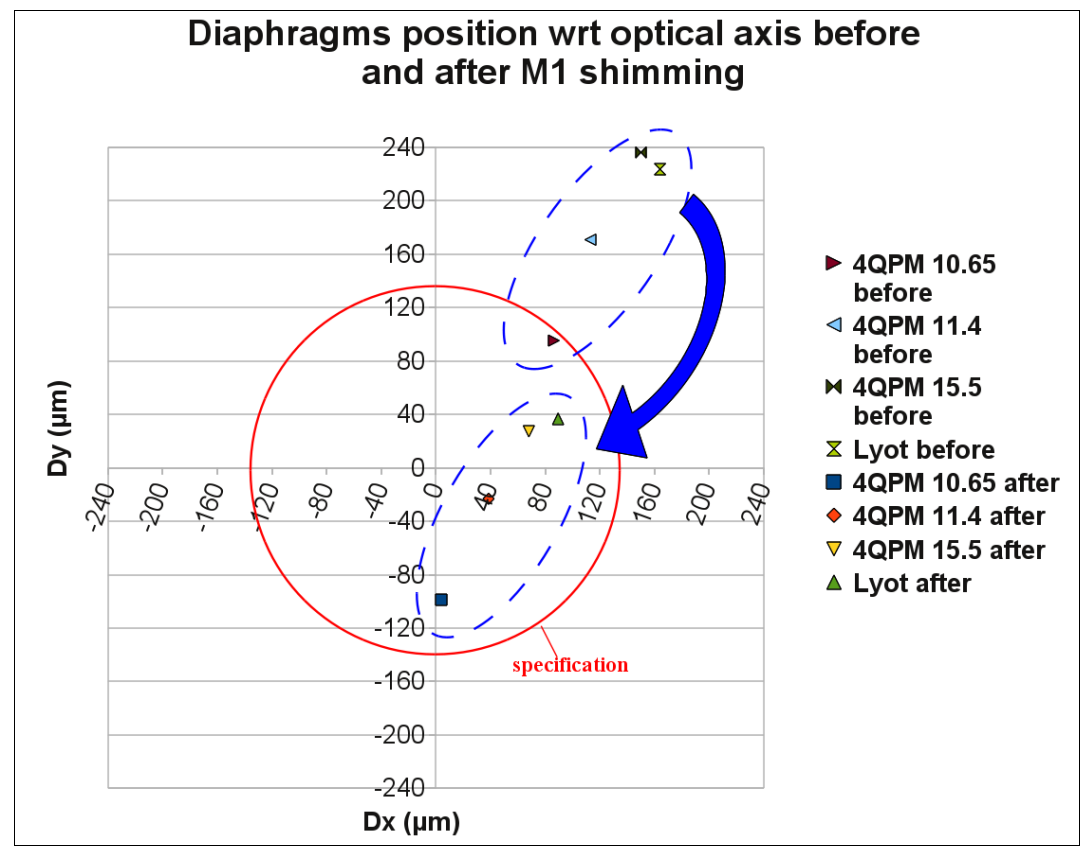

Figure 7. Alignment of the coronagraphic diaphragms. Positions of the diaphragms with respect to the optical axis are measured before and after M1 shimming. The process brings all the diaphragms center within the specification. 
Table 1: Final diaphragm positions measured at room temperature. Uncertainty is $+/-23 \mu \mathrm{m}$. Allocated budget is $140 \mu \mathrm{m}$ for coronagraphic diaphragms

\begin{tabular}{|l|l|l|}
\hline Diaph & $\mathbf{X}(\boldsymbol{\mu m})$ & $\mathbf{Y}(\boldsymbol{\mu m})$ \\
\hline 4QPM 10.65 & 4 & -99 \\
\hline 4QPM 11.4 & 39 & -23 \\
\hline 4QPM 15.5 & 68 & 27 \\
\hline Lyot & 89 & 37 \\
\hline
\end{tabular}

MIRIM is now aligned, and the test sequence can continue, with the cold performance tests using the telescope simulator.

\section{IMAGING PERFORMANCES}

\subsection{Overview and test set-up}

Contrary to the tests described previously, the purpose of which was to make fine adjustment in the system, the tests described below are proper measurements of the instrument performances in imaging, coronagraphy and spectroscopy modes. A warm telescope simulator was developed for this purpose. It delivers to MIRIM a beam similar to that of the JWST. An optical layout can be seen in figure 8 .

\section{Optical characteristics:}

This is a system with two powered mirrors (off-axis parabola - one for collimation, one for re-imaging). A point source is re-imaged and focused at MIRIM entrance focal plane with a magnification of -1 . The simulator has a pupil stop that is conjugated with MIRIM entrance pupil plane and reproduces the shape of the JWST pupil. It covers a field of $80 \times 80 \mathrm{~mm}^{2}$ with a diffraction limited optical quality. The pupil stop is mounted on a translation stage with 4 degrees of freedom to simulate misalignment of the pupils (pupil shear), to which the coronagraphic observations are very sensitive.

\section{Stability}

The coronagraphy mode drives the specifications in terms of stability of the bench. An image of the point source must be stable within $+/-3 \mu \mathrm{m}$ during 30 minutes. For this purpose, the whole system (telescope simulator, sources, cryostat) is mounted on 2 optical benches tightly linked together and mounted themselves on anti-vibration pads. To avoid slow drift due to thermal variation, the room is equipped with air conditioning and temperature control.

\section{Sources}

The telescope simulator provides illumination to MIRIM entrance focal plane with either an extended source or a pointlike source. The extended source is a $100 \times 100 \mathrm{~mm}^{2}$ flat black body with a surface temperature range of $-5^{\circ} \mathrm{C}$ to $+100^{\circ} \mathrm{C}$. The uniformity is better than $0.3^{\circ} \mathrm{C}$, with a temperature measurement accuracy of $0.3^{\circ} \mathrm{C}$ and the emissivity is 0.98. Again, the point source specifications are driven by the need for coronagraphy. The source is a LOT-ORIEL ceramic element with a temperature up to $2000 \mathrm{~K}$. The light is coupled to a $30 \mu \mathrm{m}$ pinhole. For tests less regarding in terms of source size and demanding more throughput, the pinhole can be changed for a larger one $(50 \mu \mathrm{m}, 80 \mu \mathrm{m}$...up to $1 \mathrm{~mm})$. The source is mounted on a hexapod remotely controlled, so it can be placed anywhere in the field of view of MIRIM with an accuracy of a few tens of microns. Within a short range $(1 \mathrm{~mm})$, the source can even be moved with a higher accuracy (a few $\mu \mathrm{m}$ ), which is a great advantage for fine calibration of the sensitivity to pointing errors of the coronagraphic observation, or for micro-scanning of PSF.

\subsection{PSF at all wavelengths}

A PSF was measured in each imager filter. In figure 9, we show an example of three PSFs in filters at 7.7, 12.8 and $15.0 \mu \mathrm{m}$. Signal to noise ratio is more favourable at $7.7 \mu \mathrm{m}$ (source emissivity, atmospheric and cryostat optics transmission are better), so diffraction pattern is clearly visible. One can also see the ghost reflection (about 20 pixels below the main PSF) that is due to the reflection on the cryostat entrance window (this is not a MIRIM feature). 


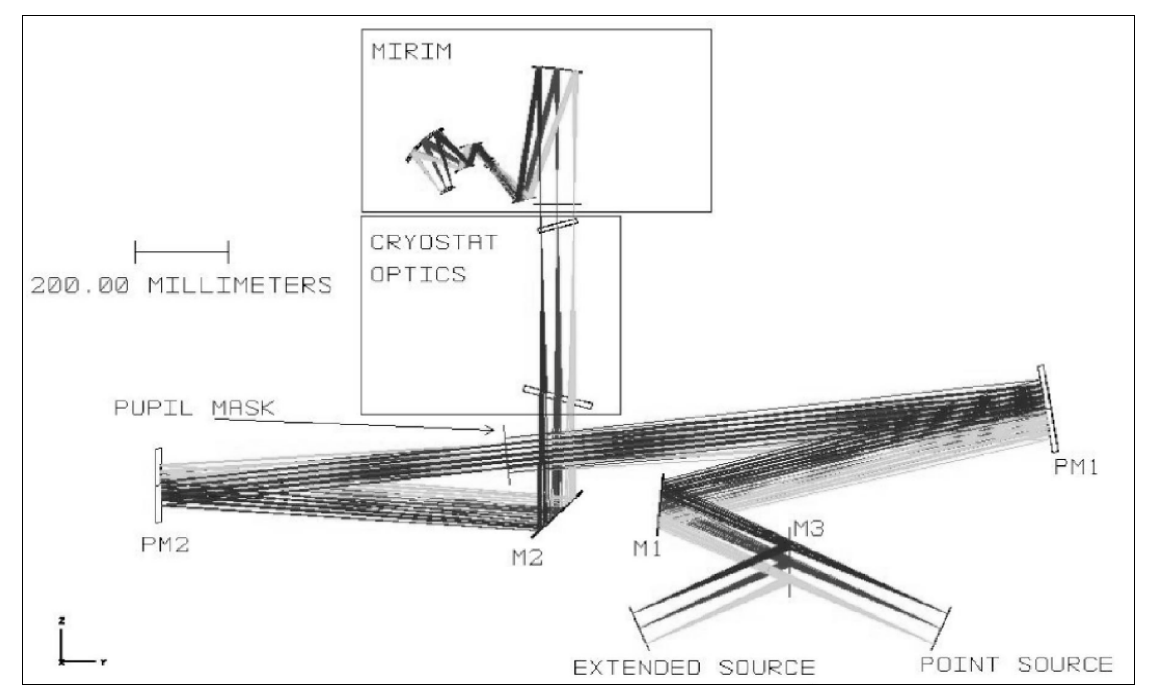

Figure 8. optical layout of the telescope simulator and MIRIM inside the cryostat. Either the extend source or the point source is imaged at MIRIM entrance focal plane via two off-axis parabola PM1 and PM2, and a set of flat mirrors. The pupil mask reproduces the JWST pupil and is adjustable with 4 degrees of freedom (three translations and one rotation around the optical axis), allowing fine alignment with MIRIM and simulation of pupil shear.

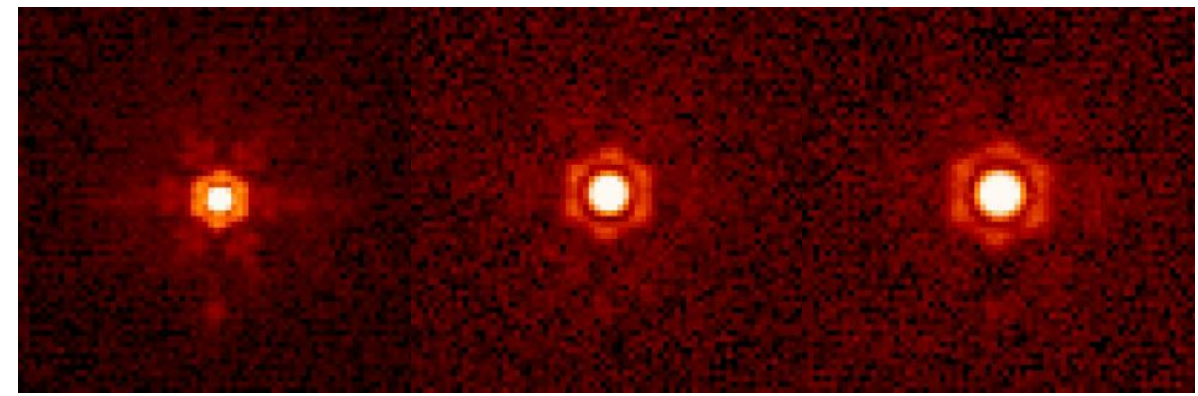

Figure 9. Three PSFs of MIRIM FM at 7.7, 12.8 and $15.0 \mu \mathrm{m}$, from left to right respectively

This time, the test is as close as we can to the real conditions of observations that MIRIM will have in the JWST. We can then compare our results directly with the requirements. Requirement on image quality tells us that PSF shall include more than $56 \%$ of the encircled energy of the image of a point source at wavelengths longward of $5.6 \mu \mathrm{m}$ within the angular diameter of the first dark ring. The encircled energy is defined relative to the energy within a radius of 5 arcsec equivalent projected onto the sky. The results of such a measurement are given in table 2 and figure 10.

Table 2. Amount of energy within first dark ring radius at various wavelengths

\begin{tabular}{|l|l|l|l|l|l|l|l|l|}
\hline Filter & F770W & F1130W & F1280W & F1500W & F1800W & F2100W & F2550W & F2550WR \\
\hline Wavelength $(\boldsymbol{\mu m})$ & 7.7 & 11.3 & 12.8 & 15.0 & 18.0 & 21.0 & 25.5 & 25.5 \\
\hline $\begin{array}{l}\text { Radius of 1 } \mathbf{1}^{\text {st }} \text { dark ring } \\
\text { (arcsec on sky) }\end{array}$ & 0.29 & 0.43 & 0.49 & 0.57 & 0.69 & 0.80 & 0.97 & 0.97 \\
\hline Encircled energy & $60.1 \%$ & $61.8 \%$ & $62.0 \%$ & $67.9 \%$ & $58.9 \%$ & $63.3 \%$ & $60.4 \%$ & $60.9 \%$ \\
\hline
\end{tabular}

The requirement given above is met at all wavelengths above $7.7 \mu \mathrm{m}$. At the shortest wavelength (5.6 $\mu \mathrm{m})$, the PSF is not Nyquist sampled. For this reason, its study is the scope of another test where we use the ability of the telescope simulator to finely scan the PSF on the grid of pixels in order to reconstruct a PSF with enhanced resolution, or sub-pixel resolution, as described in the next section. 

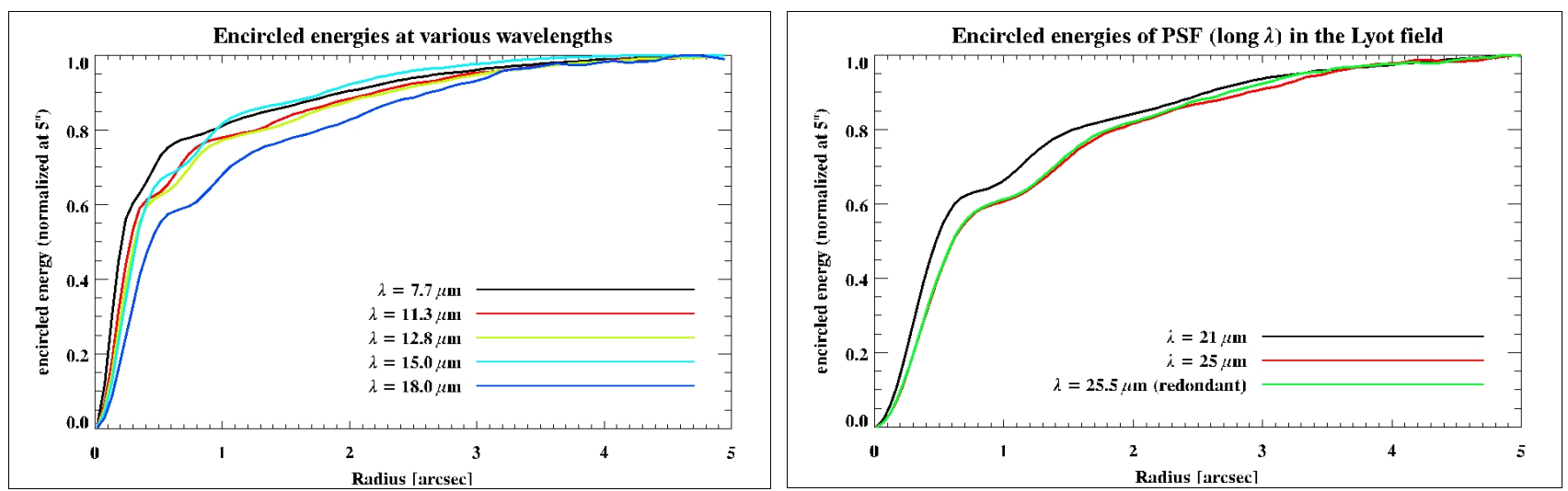

Figure 10. Left: Encircled energy of PSF at $7.7 \mu \mathrm{m}, 11.2 \mu \mathrm{m}, 12.8 \mu \mathrm{m}, 15.0 \mu \mathrm{m}$ and $18 \mu \mathrm{m}$, at the centre of the field of view.

Right: Encircled energy of PSFs in filters at $21 \mu \mathrm{m}$ and $25.5 \mu \mathrm{m}$, in Lyot field of view. X-axis scale is micrometer from the PSF centroid. Y-axis is encircled energy normalized at a distance of $5 \operatorname{arcsec}(1.11 \mathrm{~mm}$ on the detector)

\subsection{Microscan}

The goal of the micro-scanning test is to obtain an over-resolved image of the PSF at 5.6um from observed multiple lowresolution (LR) images, to perform an accurate measurement of the image quality at the shortest operating wavelength. The methodology and results of this test are described in Guillard et $a l^{[3]}$. We summarize here their main results

Figures 11 shows a simulated PSF, the PSF observed without any enhancement, and the high-resolution reconstructed PSF. Guillard et a $l^{[3]}$ showed that encircled energies and profile of PSF are in very good agreement with the simulation.
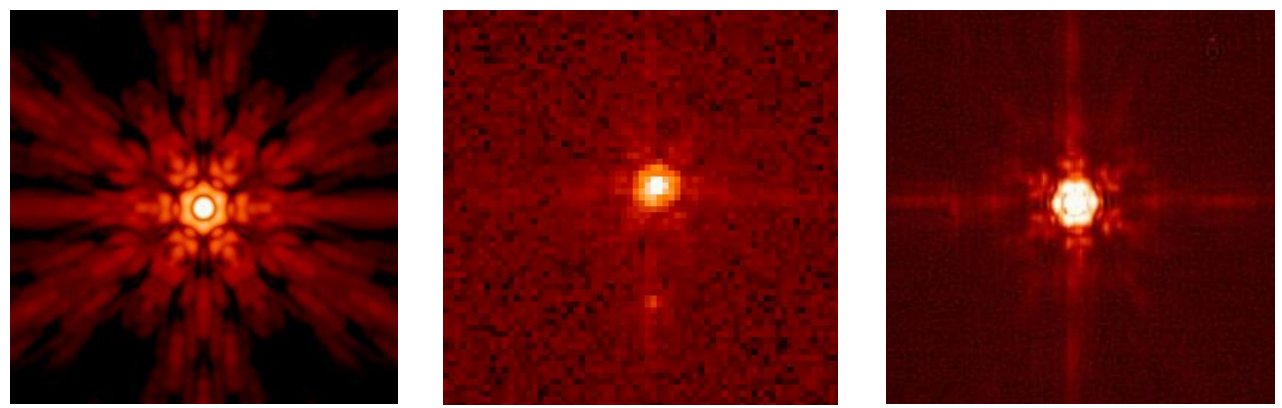

Figure 11: left: simulated PSF at $5.6 \mu \mathrm{m}$ from Zemax. Pixel size is $3.125 \mu \mathrm{m}$. Middle: raw PSF observed at $5.6 \mu \mathrm{m}$. Pixel size is $25 \mu \mathrm{m}$. Right: reconstructed PSF from microscan test at $5.6 \mu \mathrm{m}$. Pixel size is $3.5 \mu \mathrm{m}$

\section{CORONAGRAPHY}

\subsection{Overview}

Coronagraphy is the observation technique by which the light of a bright punctual object (typically, a star) is suppressed in order to allow observation of a fainter object in its neighbourhood (companion, disk...). MIRIM has two types of coronagraphs. A classical Lyot coronagraph at $23 \mu \mathrm{m}$ uses a mask in the focal plane that is a simple opaque disk that stops the light over a diameter of $6 \lambda / \mathrm{D}$. Three four-quadrant phase mask coronagraphs at $10.65,11.4$ and $15.5 \mu \mathrm{m}$ phase-shift only some areas of the PSF by $\pi$ to lead to a very specific distribution of the energy in the pupil plane. Both type of coronagraphic masks are associated to a diaphragm in the pupil plane to optimise the filtering of the light coming from the object to attenuate. More details will be found in Rouan et al. ${ }^{[4]}$.

Performance of the coronagraph is quantified by its rejection. Maximum rejection is the ratio between the maximum of the star (outside the coronagraph) and the maximum of the coronagraphic image (image obtained with the star centred on the coronagraph). The total rejection is the total illumination of the star divided by the total of the coronagraphic image. Attenuation is the measure of the maximum rejection rate as a function of the distance of the star from the centre of the coronagraph. We can then derive another quantity to estimate the performances of the coronagraph: the inner working 
angle (hereafter IWA), defined as the angular separation from the centre of the coronagraphic mask for which the attenuation is $50 \%$ of the maximum attenuation obtained with a well centred star. One of the great interest of the $4 \mathrm{QPM}$ coronagraph lies in their small IWA.

Because of the high background level due to the warm telescope simulator, the coronagraphic tests do not permit verification of the requirements, expressed in term of total rejection and contrasts at different angular separations. Here, the evaluation of performances of the system is made by comparing the test results with simulated performance of the system in comparable conditions.

These simulations are as representative as possible of real conditions:

- the entrance pupil is that of the simulator (similar to that of the JWST without segmentation).

- the pinhole is not punctual but has a physical size (almost $30 \mu \mathrm{m}$ in diameter at MIRIM entrance focal plane,

except for the Lyot coronagraph where it is $40 \mu \mathrm{m}$ ).

- the coronagraph is simulated with real mask and real diaphragm.

- theoretical spectral resolution of the filter is used (20 for Four Quadrant Phase Mask coronagraphs, 5 for the

Lyot coronagraph).

Results of coronagraphic tests on previous model of MIRIM are described in Cavarroc et al. ${ }^{[5]}$.

\subsection{Results}

A raster pattern is defined to scan the PSF around the centre of the mask. The aim is to study the shape of the PSF for different positions in the field of view. Linear scan of the PSF are also used to determine the attenuation as a function of the distance to the centre of the mask.

Data acquisition is made with a pinhole of $30 \mu \mathrm{m}$ for the $10.65 \mu \mathrm{m}$ and $11.4 \mu \mathrm{m}$ coronagraphs, and $40 \mu \mathrm{m}$ for the $15.5 \mu \mathrm{m}$, which is about 8 times smaller than the FWHM of the diffraction pattern.

The effect of the coronagraph is illustrated in figure 12 below. On the coronagraphic image, residual light is observed, in the shape of a extended and faint source. This is due to the surrounding of the pinhole that is warmed up by the source, and it limits the maximum rejection attainable. Figure 13 shows the profiles of both images (PSF and coronagraphic). One can see that measured profiles and simulated ones are compliant.

Inner working angle measurements are made by scanning the PSF across the centre of the quadrant-phase mask and measuring the rejection on the maximum. It is then possible to plot an attenuation curve. The full width at half-maximum of this curve is the IWA. Figure 14 shows attenuation curves for various scans. IWA measured is $1.3 \lambda / \mathrm{D}$ for the scan passing through the centre of the mask. The expected value from simulations is $1.2 \lambda / \mathrm{D}$.

The different results of the measurements are listed in table 3 . The rejection measured on the 4QPM are slightly smaller than what was expected. This is mainly due to the residual light coming from the warmed up metal around the pinhole, as explained above.

Table 3. Main results from the coronagraphic tests on quadrant-phase mask and Lyot coronagraphs.

\begin{tabular}{|c|c|c|c|c|c|}
\hline $\begin{array}{c}\text { Rejection on } \\
\text { maximum } \\
(10.65 \mu \mathrm{m})\end{array}$ & $\begin{array}{c}\text { Inner Working } \\
\text { Angle } \\
(10.65 \mu \mathrm{m})\end{array}$ & $\begin{array}{c}\text { Rejection on } \\
\text { maximum } \\
(11.4 \mu \mathrm{m})\end{array}$ & $\begin{array}{c}\text { Rejection on } \\
\text { maximum } \\
(15.5 \mu \mathrm{m})\end{array}$ & $\begin{array}{c}\text { Rejection on } \\
\text { maximum } \\
(\text { Lyot } 23 \mu \mathrm{m})\end{array}$ & $\begin{array}{c}\text { Inner Working } \\
\text { Angle }(23 \mu \mathrm{m})\end{array}$ \\
\hline 173 & $1.3 \lambda / \mathrm{D}$ & 330 & 250 & 60 & $2.5 \lambda / \mathrm{D}$ \\
\hline
\end{tabular}

\subsection{Conclusion on the coronagraphic tests}

The optical bench has been very powerful to test the coronagraphic mode of MIRIM. The small size of the pinhole, the accurate movement of the source, the mechanical stability of the whole bench allowed a very good assessment of the performances. The main limitation is brought by the high background of the telescope simulator at room temperature, but the performances measured are close to the expected ones in such observational conditions. 

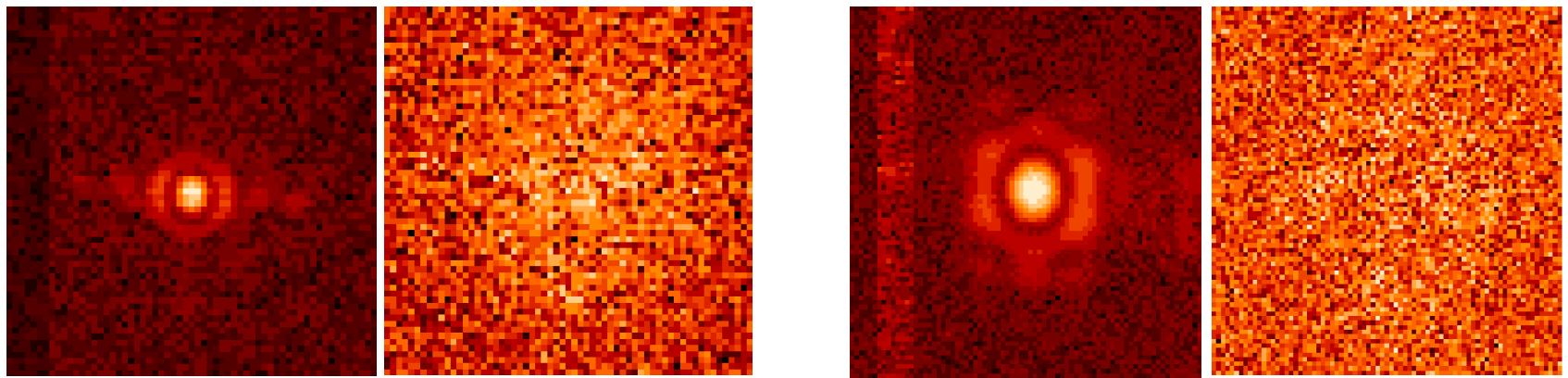

Figure 12. Left: PSF at $10.65 \mu \mathrm{m}$, far from the centre of the coronagraphic mask and the corresponding coronagraphic image, obtained with the the same PSF centred on the mask. Right: same observations for the Lyot coronagraph at $23 \mu \mathrm{m}$.
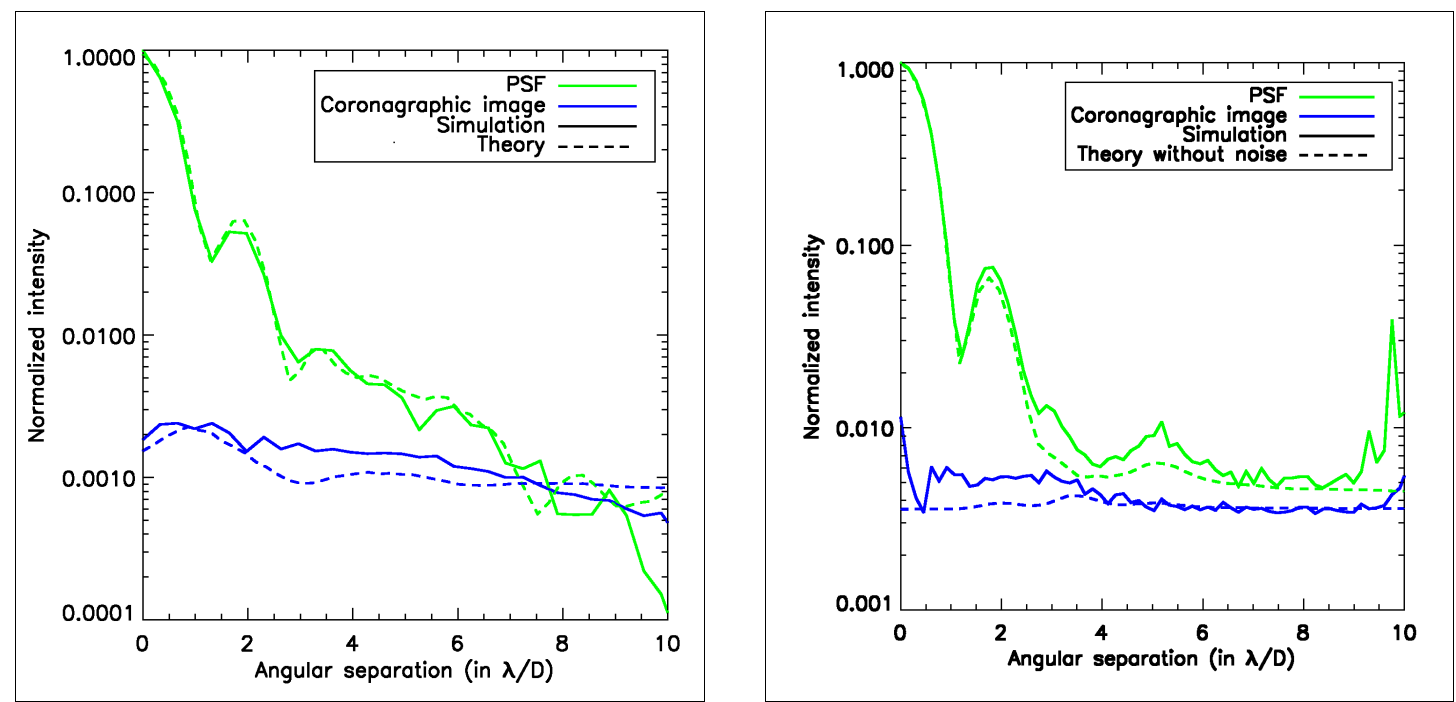

Figure 13. Normalized coronagraphic profile and PSF (plain lines) compared to simulated profiles (dash lines) for a the 4QPM coronagraph at $10.65 \mu \mathrm{m}$ (left) and the Lyot coronagaph at $23 \mu \mathrm{m}$ (right)

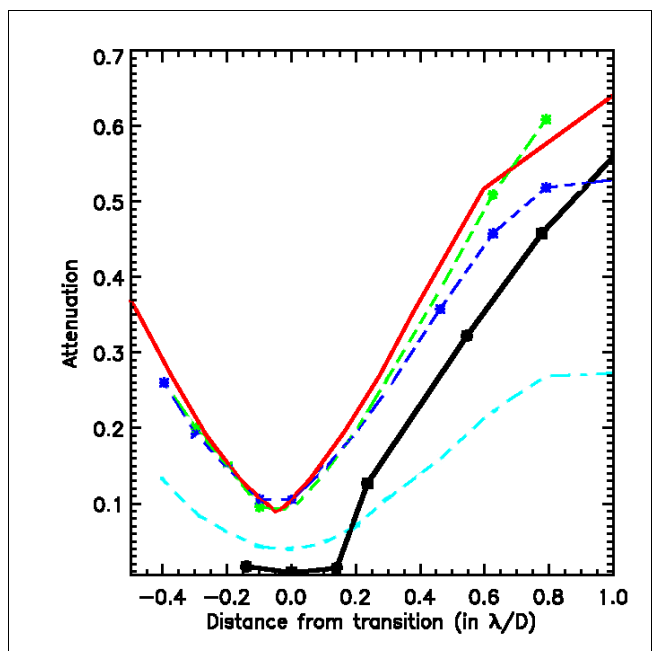

Figure 14: Attenuation as a function of the distance to the centre of the coronagraphic mask. The thick dark line corresponds to a scan of the source through the centre of the mask (other curves are for different scans not through the centre) 


\section{LOW RESOLUTION SPECTROSCOPY}

In order to perform low resolution spectroscopy (LRS) in MIRIM, the filter wheel is fitted with a double prism in the pupil plane and a 0.6 " wide slit is mounted in the entrance focal plane. The LRS covers the 5-10 $\mu \mathrm{m}$ range.

The test plan on the LRS includes wavelength calibration and measurement of spectral power at $7.5 \mu \mathrm{m}$. Specifications state that the wavelengths in the MIRI low resolution spectra shall be known, after ground data processing and application of calibration data, to within $10 \%$ full width half maximum of the spectral resolution element for an unresolved line. As for the resolving power, it shall be $100+/-10$ at the wavelength of $7.5 \mu \mathrm{m}$.

\subsection{Wavelength calibration}

\section{Test overview}

A broad-band spectrum was produced with the LRS using a blackbody point source. As the source is located several metres outside the instrument cryostat, the spectrum is marked by the distinctive absorption features from water in the humid air. In addition, several polycarbonate filters were inserted into the path in a number of combinations, to provide further spectral markers to be used in the wavelength calibration. The filters were measured to high precision in a vacuum with a Fourier transform spectrometer. The atmospheric transmission profile over the LRS wavelength range was modelled using the BTRAM radiative transfer model ${ }^{[6]}$.

\section{Method of analysis}

The LRS spectral data were flat-fielded, linearised and background subtracted. Spectra were centroided and extracted along a 7-pixel column along the length of the detector subarray (see figure 15 left). The optical model as produced in Zemax was applied to the measured spectrum as a starting estimate for the pixel-wavelength conversion.

A model of the input signal was calculated using the specifications (temperature, emissivity) of the blackbody source; the atmospheric transmission spectrum; and filter transmission profiles as appropriate. This profile was used as the test metric for the LRS wavelength calibration (see figure16 right). Gaussian fits to distinct spectral features allowed us to estimate the quality of the fit (figure 15 right). The optical model provided an excellent fit to the model spectrum within the central region of the LRS range (6-8 um), but outside this range some inaccuracy remains. Figure 16 left shows the difference in modelled and retrieved wavelength in the spectra from different filter combinations, compared against the requirement.

The fitting procedure is complicated by the complex non-linear shape of the LRS dispersion relation and the lack of unresolved and unblended spectral lines at $\mathrm{R}$ of 100 , outside of the 6-8 um range, to improve the fit. Several crosscorrelation fine-tuning methods were tested, but none of these significantly improved the quality of the wavelength solution over the full spectral range. Additional tests will be performed in the full-system testing in 2011 to improve the wavelength calibration for the LRS.

\subsection{Resolving power}

\section{Test overview}

The spectral resolving power of LRS was estimated using a monochromator at the input of the telescope simulator. It provides a quasi-monochromatic point source, tunable between 4 and $15 \mu \mathrm{m}$. The monochromator was stepped from 7 to $8 \mu \mathrm{m}$ in $0.1 \mu \mathrm{m}$ shifts, to measure in detail the change in line width with wavelength.

\section{Analysis}

The spectra were flat-fielded, background subtracted and extracted along the brightest pixel. Gaussians were fitted to each line, and the FWHM calculated from the fitted sigma value using: $2 \sqrt{2 \ln (2)} \times \sigma$. The error on the FWHM was calculated from the error on sigma, typically $\sim 10 \%$.

The line width is estimated to be $\sim 9 \mathrm{~nm}$ at $7.5 \mu \mathrm{m}$ ( $\mathrm{R} \sim 800$ ), and this intrinsic width was subtracted from the measured linewidth in quadrature to recover the intrinsic LRS resolving power.

The resulting resolving power trend between 7 and $8 \mu \mathrm{m}$ is shown in figure 18. At $7.5 \mu \mathrm{m}, \mathrm{R}$ was calculated to be 95.3 $+/-0.6$, within the as-designed specification. 

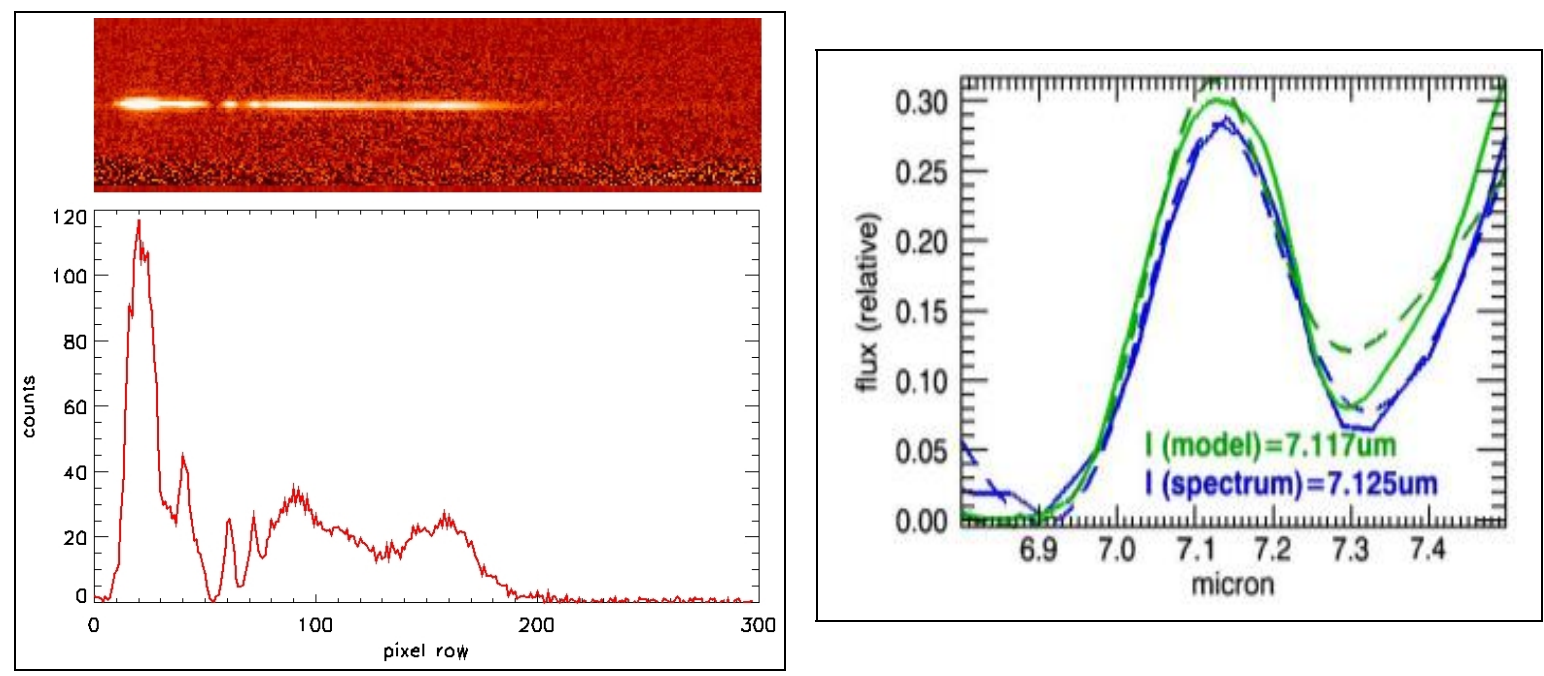

Figure 15: Top left: spectrum of the black body source observed with the LRS of MIRIM. The spectrum is modulated by absorption features found in atmosphere and various filters put in the optical path. Bottom left: the corresponding profile, extracted along a 7-pixel wide line centered on the maximum. Right: detail of a feature showing a Gaussian fit of model spectrum and measured one, after fine tuning wavelength calibration
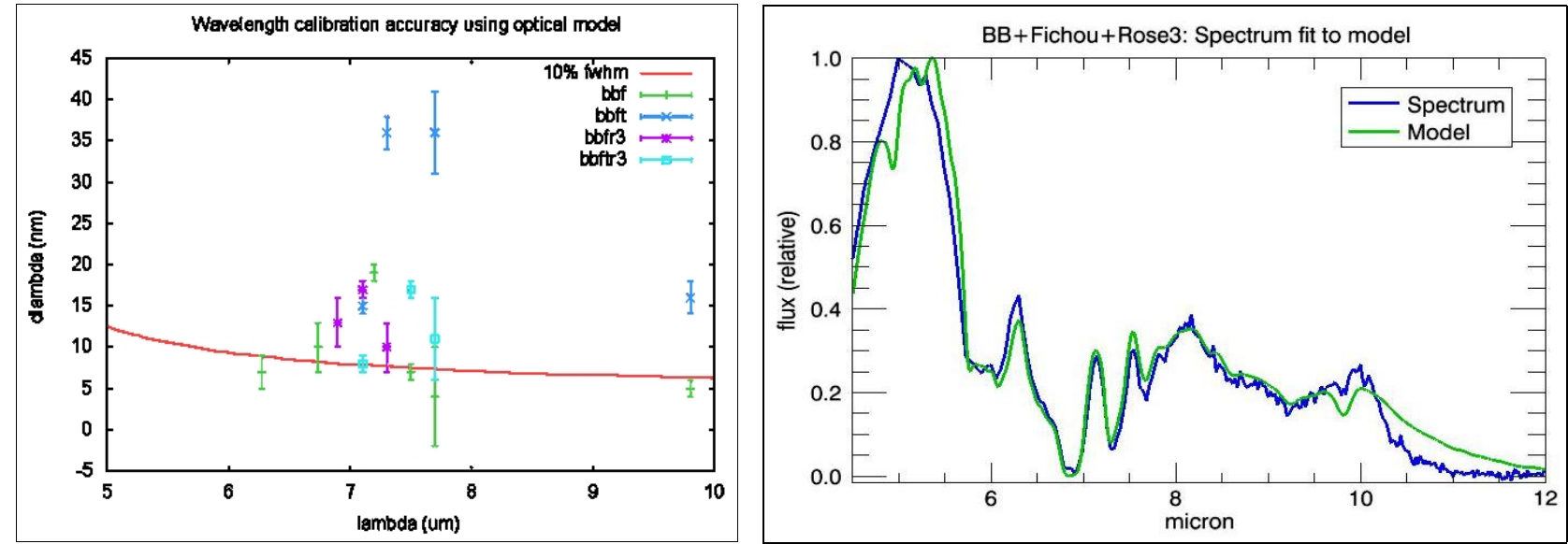

Figure 16: Left: Differences between the LRS spectrum and the calculated input spectrum, using Gaussian fits of distinctive features in the spectra and comparing Gaussian centres. Different colours represent different filter combinations. The differences are expressed in term of $\mathrm{d} \lambda$, in nanometre. Right: Measured spectrum over-plotted with source model, after wavelength calibration.

\section{CONCLUSION}

In this paper, we have presented the main results of the test campaign on the MIRIM flight model at CEA. A lot of data have been gathered, and some of them are unique in the test program. The main results are summarized below:

- Spot sizes were measured at ambient and cold temperatures, in the whole field, and were found to be satisfactory. Position of best focus was accurately measured, and detector mounted accordingly.

- Magnification and distortion were measured at ambient and cold temperatures and are satisfactory. A map giving the amount of distortion in the whole field have been computed.

- Alignment of the coronagraphic diaphragms have been performed at ambient temperature and was checked at cold temperature. All diaphragms positions are now within specification. 

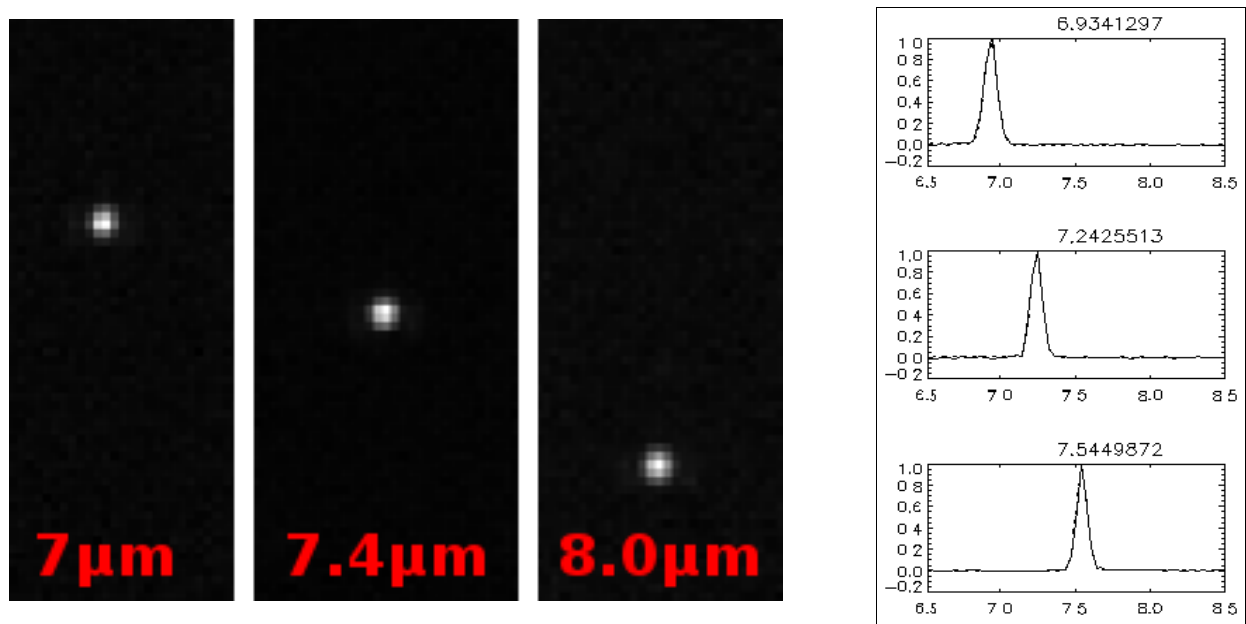

Figure 17: Left: illustration of the movement of the source on the detector as the wavelength is scanned between 7 and $8 \mu \mathrm{m}$. Right: Profile of three of the eleven lines used for measurement of the resolving power. There are 11 lines between 7 and $8 \mu \mathrm{m}$ by step of $0.1 \mu \mathrm{m}$. Gaussian profile was fitted to each line.

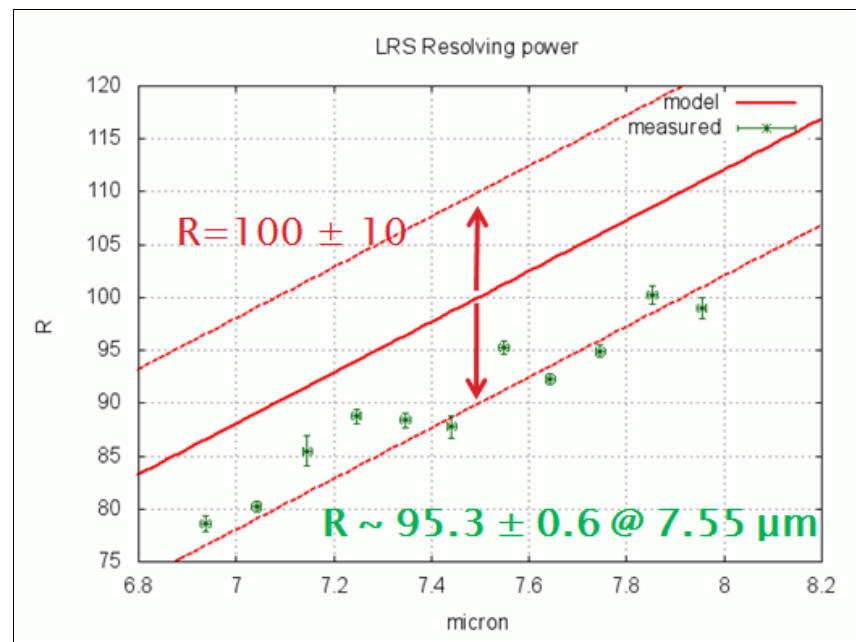

figure 18: measured resolving power at a function of wavelength. Resolving power at $7.54 \mu \mathrm{m}$ is $94.06+/-0.6$

- Point spread functions have been measured at all wavelengths, at least at the centre of the field of view, and in the corners for some of them. They all are compliant with the Zemax simulations.

- PSF at $5.6 \mu \mathrm{m}$ have been measured in the whole field of view using a micro-scanning technique, which provide a more accurate characterization, and was found to be compliant with the simulations. More details can be found in Guillard et al., 2010 $0^{[3]}$.

- Coronagraphs have all been thoroughly tested and appeared to perform in accordance with simulation. Limitation to reach the theoretical rejection is due to the high background of the warm telescope simulator.

- The low resolution spectroscopy mode have been characterized. A wavelength calibration was made, and the resolving power at $7.5 \mu \mathrm{m}$ have been measured and is within specification.

The next stage in MIRIM's life on ground is its delivery to the Rutherford Appleton Laboratory (RAL, UK), where it is assembled with the spectrometer, to form the whole instrument MIRI. It will be again extensively tested in even more "flight-like" conditions (optical train includes the external input optics and calibration unit, and the telescope simulator is cold).

With the unique capabilities of the test bench in terms of fine-scanning, stability, and small size and mono-chromaticism of the source, the test program at CEA allowed to perform a complementary and very valuable characterization of MIRIM. 


\section{REFERENCES}

[1] Wright, G. S., Rieke, G. H., Colina, L., van Dishoeck, E., Goodson, G., Greene, T., Lagage, P.-O., Karnik, A., Lambros, S. D., Lemke, D., Meixner, M., Norgaard, H.-U., Oloffson, G., Ray, T., Ressler, M., Waelkens, C., Wright, D., and Zhender, A., "The JWST MIRI instrument concept," SPIE 5487, 653-663 (2004).

[2] Amiaux, J., Alouadi, F., Augueres, J. L., Bouchet, P., Bouzat, M., Cavarroc, C., Cloue, C., De Antoni, P., Desforges, D., Donati, A., Dubreuil, D., Eppelle, D., Gougnaud, F., Hervieu, B., Lagage, P. O., Leboeuf, D., Le Mer, I., Lussignol, Y., Mattei, P., Meigner, F., Moreau, V., Pantin, E., Perrin, P., Ronayette, S., Tauzin, G., Poupar, S., Wright, D., Glasse, A., Wright, G., Mazy, E., Plesseria, J. Y., Renotte, E., Ray, T., Abergel, A., Guillard, P., Longval, Y., Ressler, M., Reess, J. M., Hofferbert, R., Krause, O., Justtanont, K., and Olofsson, G., "Development approach and first infrared test results of JWST/Mid Infra Red Imager Optical Bench,” SPIE 7010, (2008).

[3] Guillard P., Rodet T, Ronayette S., Amiaux J., Abergel A., Moreau V., Augueres J.L., Bensalem A., Orduna T., Nehmé C., Belu A. R., Pantin E., Lagage P.-O., Longval Y., Glasse A. C. H., Bouchet P., Cavarroc C., Dubreuil D., Kendrew S., "Optical performance of the JWST/MIRI flight model: characterization of the point spread function at high-resolution," SPIE 7731, (2010).

[4] D. Rouan, P. Riaud, A. Boccaletti, Y. Clénet, and A. Labeyrie, “The Four-Quadrant Phase-Mask Coronagraph. I. Principle," PASP 112, 1479-1486, (2000)

[5] C. Cavarroc, J. Amiaux, P. Baudoz, A. Boccaletti, D. Dubreuil, P.-O. Lagage, V. Moreau, P. Bouchet, E. Panin, J.-M. Reess; S. Ronayette; G. S. Wright, "First tests of the coronagraphic device of MIRI/JWST," SPIE 7010, (2008).

[6] Davis-Imhof, P., “BTRAM,” http://blueskyspectroscopy.com/BlueSkyInc/TopLevelPages/BTRAM.html, (2010) 